\title{
Nanoscale Near-Field Tomography of Surface States on $\left(\mathrm{Bi}_{0.5} \mathrm{Sb}_{0.5}\right)_{2} \mathrm{Te}_{3}$
}

\author{
Fabian Mooshammer, ${ }^{\dagger}{ }^{\circ}$ Fabian Sandner, ${ }^{\dagger}$ Markus A. Huber, ${ }^{*}{ }^{\dagger}$ Martin Zizlsperger, ${ }^{\dagger}$ Helena Weigand, \\ Markus Plankl, ${ }^{\dagger}$ Christian Weyrich, ${ }^{\ddagger}$ Martin Lanius, Jörn Kampmeier, ${ }^{+}$Gregor Mussler, \\ Detlev Grützmacher, Jessica L. Boland, ${ }^{\dagger}{ }^{\ddagger}$ Tyler L. Cocker, ${ }^{\S}$ and Rupert Huber ${ }^{*}{ }^{\dagger}$ \\ ${ }^{\dagger}$ Department of Physics, University of Regensburg, 93040 Regensburg, Germany \\ ${ }^{\ddagger}$ Peter Grünberg Institut 9, Forschungszentrum Jülich \& JARA Jülich-Aachen Research Alliance, 52425 Jülich, Germany \\ ${ }^{\S}$ Department of Physics and Astronomy, Michigan State University, East Lansing, Michigan 48824, United States
}

\section{Supporting Information}

\begin{abstract}
Three-dimensional topological insulators (TIs) have attracted tremendous interest for their possibility to host massless Dirac Fermions in topologically protected surface states (TSSs), which may enable new kinds of high-speed electronics. However, recent reports have outlined the importance of band bending effects within these materials, which results in an additional two-dimensional electron gas (2DEG) with finite mass at the surface. TI surfaces are also known to be highly inhomogeneous on the nanoscale, which is masked in conventional far-field studies. Here, we use near-field microscopy in the midinfrared spectral range to probe the local surface properties of custom-

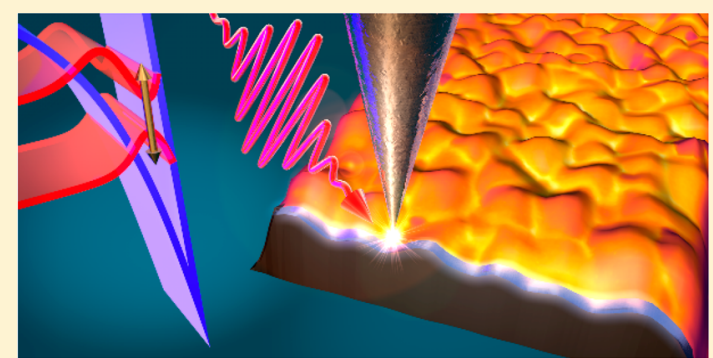
tailored $\left(\mathrm{Bi}_{0.5} \mathrm{Sb}_{0.5}\right)_{2} \mathrm{Te}_{3}$ structures with nanometer precision in all three spatial dimensions. Applying nanotomography and nanospectroscopy, we reveal a few-nanometer-thick layer of high surface conductivity and retrieve its local dielectric function without assuming any model for the spectral response. This allows us to directly distinguish between different types of surface states. An intersubband transition within the massive $2 \mathrm{DEG}$ formed by quantum confinement in the bent conduction band manifests itself as a sharp, surface-bound, Lorentzian-shaped resonance. An additional broadband background in the imaginary part of the dielectric function may be caused by the TSS. Tracing the intersubband resonance with nanometer spatial precision, we observe changes of its frequency, likely originating from local variations of doping or/and the mixing ratio between $\mathrm{Bi}$ and $\mathrm{Sb}$. Our results highlight the importance of studying the surfaces of these novel materials on the nanoscale to directly access the local optical and electronic properties via the dielectric function.
\end{abstract}

KEYWORDS: Near-field microscopy, SNOM, tomography, topological insulator, surface state, band bending

$\mathrm{T}$ hree-dimensional (3D) topological insulators ${ }^{1-5}$ (TIs) have emerged as a fascinating class of solids due to their unique surface properties. Whereas the bulk material is insulating, topologically protected, metallic states are found at the surface that host massless Dirac Fermions. Owing to the chiral spin texture of these surface states, time-reversal symmetry prevents backscattering at the surface, providing hope for extremely high carrier mobilities that can be exploited in next-generation devices. ${ }^{6}$ Such spin-momentum locking holds further promise for spintronic applications, as the surface electric current is directly linked to a spin current. Control of these surface electrical currents has been achieved with continuous-wave light of variable polarization ${ }^{7}$ as well as by ultrafast photoexcitation. ${ }^{8-11}$ Furthermore, there is a variety of exciting physical phenomena that can be explored in 3D TIs, including lightwave-driven Dirac currents, ${ }^{11}$ Floquet-Bloch states, $^{12,13}$ Dirac surface plasmons ${ }^{14}$ or strong optical nonlinearities. ${ }^{15}$

Unfortunately, prototypical van der Waals-layered 3D TIs, such as $\mathrm{Bi}_{2} \mathrm{Se}_{3}$ or $\mathrm{Bi}_{2} \mathrm{Te}_{3}$, are inherently heavily doped $(\sim 1 \times$ $\left.10^{19} \mathrm{~cm}^{-3}\right)$, resulting in a finite conductivity of the bulk material. Recently, ternary alloys of $\left(\mathrm{Bi}_{1-x} \mathrm{Sb}_{x}\right)_{2} \mathrm{Te}_{3}(x=0 \ldots 1)$ have allowed full control of the Fermi level and even the position of the Dirac point relative to the band edges ${ }^{16,17}$ via tuning of $\mathrm{Sb}$ concentration. Importantly, the nature of the topologically protected surface states (TSSs) is retained over the entire range of $\mathrm{Sb}$ concentrations, providing a means to minimize the bulk conductance due to inherent doping in these materials, ${ }^{18}$ which otherwise obscures the properties of these surface states. Additionally, the surface of 3D TIs can be unintentionally doped either due to aging and degradation effects under ambient conditions, ${ }^{19}$ or structural defects and impurities. ${ }^{20}$ This surface doping induces band bending which can lead to quantum confinement of charge carriers at the surface. Consequently, a two-dimensional electron gas $^{20,21}$ (2DEG) forms, which gives rise to surface states of massive

Received: July 23, 2018

Revised: November 8, 2018

Published: November 13, 2018 


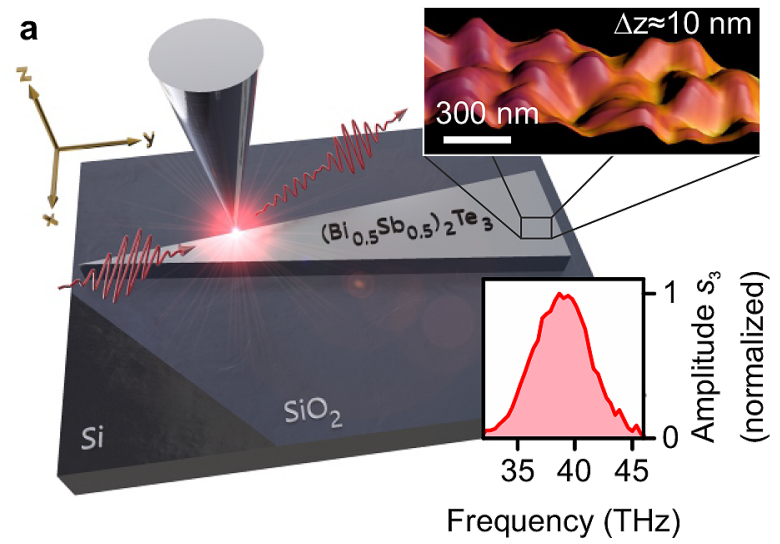

b

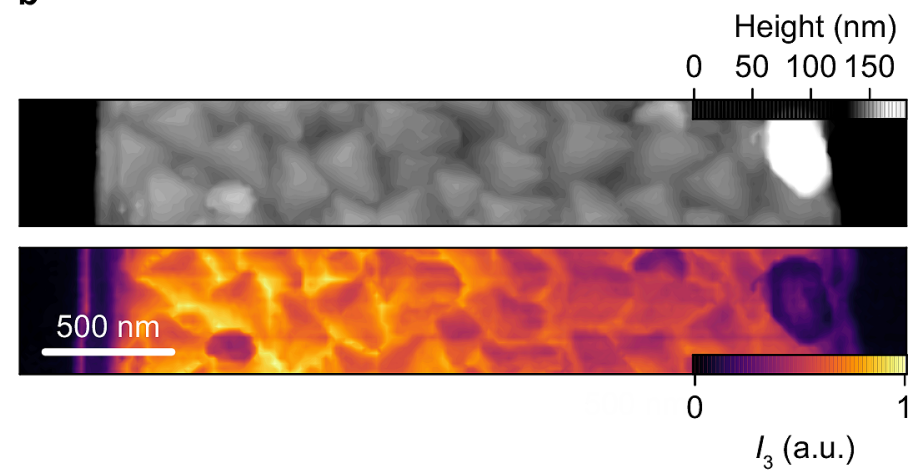

Figure 1. Schematic of near-field microscopy and the surface inhomogeneity of $\left(\mathrm{Bi}_{0.5} \mathrm{Sb}_{0.5}\right)_{2} \mathrm{Te}_{3}$ structures. (a) Schematic of the experimental setup depicting a mid-infrared pulse (red waveform) that illuminates the sharp metallic tip and is subsequently scattered back into the far field (longer, red waveform). The near fields underneath the tip apex (red shining area) probe the sample and allow the $\left(\mathrm{Bi}_{0.5} \mathrm{Sb}_{0.5}\right)_{2} \mathrm{Te}_{3}$ wedge-shaped structure to be studied on the nanoscale. The topological insulator was selectively grown on a $\mathrm{Si} / \mathrm{SiO}_{2}$ substrate with a nominal height of $\sim 80 \mathrm{~nm}$. The top inset illustrates the inhomogeneity of a typical structure showing the topography as height profile with the color-coded scattering response superimposed. The amplitude spectrum of the MIR probe pulses scattered from the near field over a silicon reference and measured interferometrically (part a, see Supporting Information for details) is shown in the bottom inset (center frequency, $38 \mathrm{THz}$; full width at halfmaximum, $6 \mathrm{THz}$ ). (b) Topography (top panel) and simultaneously acquired map of the scattered mid-infrared intensity $I_{3}$ (bottom panel) of a representative region of a $\left(\mathrm{Bi}_{0.5} \mathrm{Sb}_{0.5}\right)_{2} \mathrm{Te}_{3}$ structure.

electrons alongside the topologically protected ones. An experimental way of distinguishing these fundamentally different states is therefore essential for understanding the surface properties of TIs and designing TI-based devices that are robust under ambient conditions.

In far-field studies, the most direct way to access surface states is angle-resolved photoemission spectroscopy $^{4,9,11-13,16-18,20-24}$ (ARPES). However, this technique averages over nanoscale inhomogeneities, losing vital local information. TI surfaces possess inhomogeneity not only in their morphology, ${ }^{25}$ but also in their local electronic properties $^{26}$ and carrier density, ${ }^{27,28}$ all of which influence the properties of the surface states locally. For this reason, it is crucial to study these materials with nanometer precision to unravel the local composition of the surface states. Scanning probe techniques readily achieve such nanometer-scale resolution laterally and are therefore ideally suited to study the local properties of TI surfaces. ${ }^{25-27}$

Here, we employ scattering-type scanning near-field optical microscopy ${ }^{28-34}$ (s-SNOM) to study the surface states of $\left(\mathrm{Bi}_{0.5} \mathrm{Sb}_{0.5}\right)_{2} \mathrm{Te}_{3}$ on nanometer length scales. This technique also provides an additional tomographic sensitivity ${ }^{30,34}$ in the third spatial dimension that enables selective probing of the surface states. We identify a massive $2 \mathrm{DEG}$ and indications of the TSS. The surface states' extension is found to be on the order of $\sim 5 \mathrm{~nm}$ and their local properties are directly traced via the dielectric function. These results represent crucial insights into the inhomogeneity of TI surfaces on the nanoscale.

We investigate $\left(\mathrm{Bi}_{0.5} \mathrm{Sb}_{0.5}\right)_{2} \mathrm{Te}_{3}$ wedge-shaped structures ${ }^{35}$ that were deposited by means of molecular beam epitaxy on a prestructured $\mathrm{Si} / \mathrm{SiO}_{2}$ substrate (see Supporting Information). An $\mathrm{Sb}$ concentration of $50 \%$ was chosen, based on previously reported values, ${ }^{18}$ to ensure a maximal resistivity and a minimal density of free carriers within the bulk material, thereby reducing the response of the bulk and isolating that of the TI surface. To achieve the deeply subwavelength resolution $^{28-34,36-47}$ required to observe this response on the nanoscale, the sharp metallic tip of an atomic force microscope is placed above the sample and illuminated with broadband mid-infrared (MIR) light pulses with a center frequency of 38 $\mathrm{THz}$ and a full width at half-maximum of $6 \mathrm{THz}$ (Figure 1a, bottom inset). Underneath the tip apex, strong evanescent near fields locally probe the $\left(\mathrm{Bi}_{0.5} \mathrm{Sb}_{0.5}\right)_{2} \mathrm{Te}_{3}$ structure with a spatial resolution as good as $10 \mathrm{~nm},{ }^{30}$ overcoming the diffraction limit by orders of magnitude. The interacting tip-sample system then scatters the MIR radiation back into the far field, where it is detected using a mercury cadmium telluride semiconductor diode. By operating the metallic tip in tapping mode, we can demodulate the detected (noninterferometric) intensity $I_{\mathrm{n}}$ at harmonic orders $\mathrm{n}$ of the tapping frequency, to obtain background-free signals (see Supporting Information for experimental details). ${ }^{28-34}$

By raster scanning the tip across the sample, the topography and the scattered intensity in the MIR can be recorded simultaneously (Figure 1a, top inset), as shown in detail in Figure $1 \mathrm{~b}$ for one representative region of $\mathrm{a}\left(\mathrm{Bi}_{0.5} \mathrm{Sb}_{0.5}\right)_{2} \mathrm{Te}_{3}$ structure. Overall, the near-field intensity scattered over the TI is increased compared to the substrate. Furthermore, the topography (Figure 1b, top panel) clearly shows inhomogeneities across the surface of the ternary alloy, distinguishing triangular protrusions, which cannot be observed using far-field techniques. This nanoscale topography is also clearly discernible in the scattered intensity $I_{3}$ (Figure $1 \mathrm{~b}$, bottom panel), where the same triangular features are observed and the intensity is enhanced in the topographic troughs. This behavior may be caused either by locally enhanced near fields or by a modification of the surface states. A precise distinction of these contributions will be reserved to future studies of extremely flat samples. The scattering response in Figure $1 \mathrm{~b}$, however, exhibits an additional, larger-scale gradient, which is free of such complications and points toward local changes of the dielectric function.

As the scattered radiation probes the dielectric function close to the material surface, the global enhancement of nearfield scattering suggests that the TI surface possesses a large conductivity. We utilize nanospectroscopy to identify the type of surface states contributing to this signal. Therefore, the scattered radiation is superimposed with a MIR reference pulse 

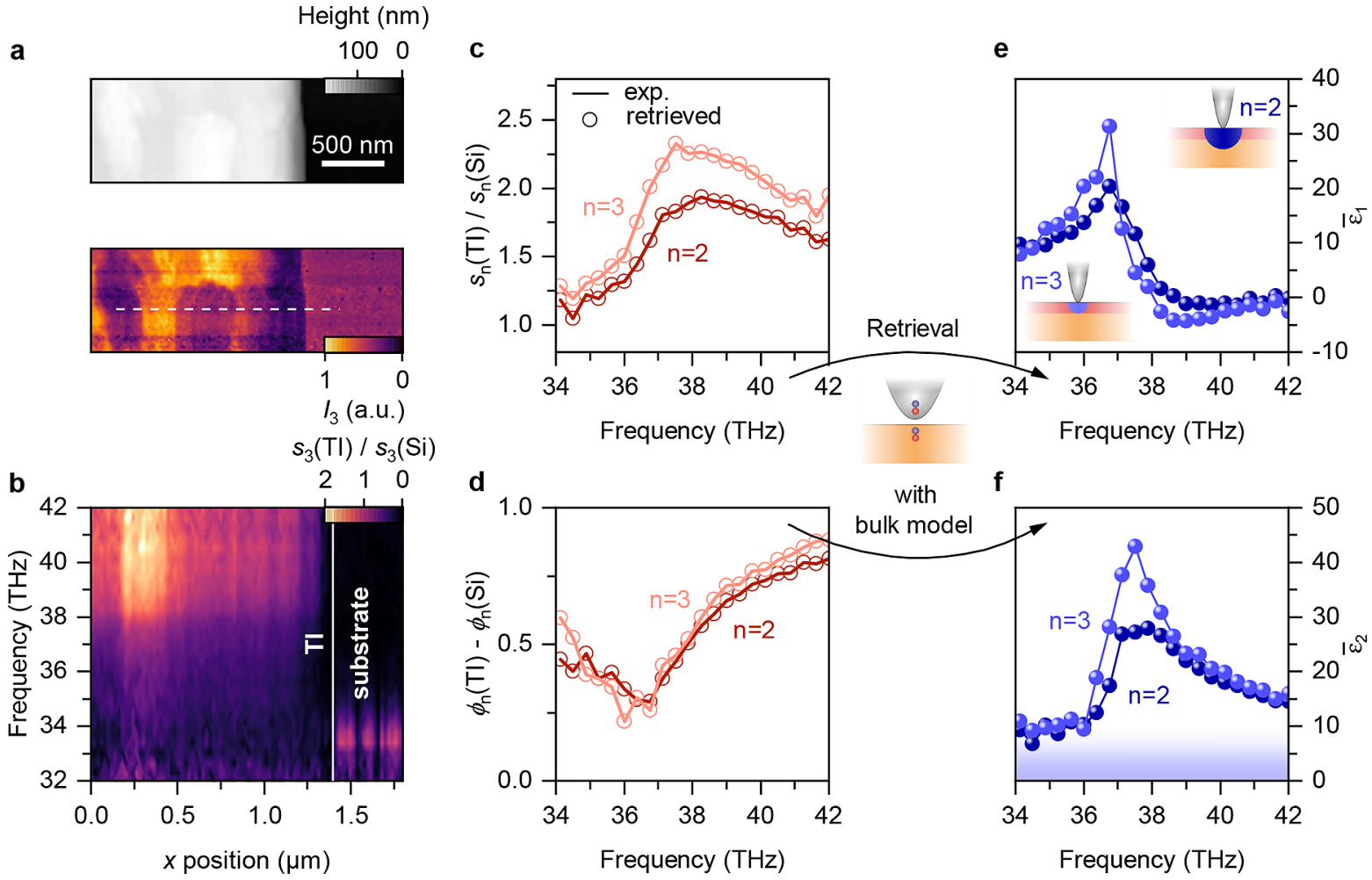

Figure 2. Spectral response of the surface state and extraction of the dielectric function. (a) Topography (top panel) and scattered intensity $I_{3}$ (bottom panel) near the edge of a TI wedge. The white dashed line indicates the path over which the tip is scanned to take the data in part b. (b) Hyperspectral measurement along the white dashed line in part a. The interferometrically measured spectral amplitude $s_{3}$ (see Supporting Information for details) normalized to the response from a silicon reference sample $s_{3}(\mathrm{Si})$ is color-coded as a function of the tip position $x$ and the probe frequency. (c, d) Scattered amplitude $s_{\mathrm{n}}(\mathrm{TI})$ and phase $\phi_{\mathrm{n}}(\mathrm{TI})$ of the topological insulator normalized to a silicon reference sample for demodulation orders $\mathrm{n}=2,3$ (solid lines). The circles indicate data points that were computed with a bulk model using the retrieved dielectric response of parts e and $\mathrm{f}$. The excellent agreement between the circles and the lines confirms the convergence of the retrieval algorithm. (e, $\mathrm{f}$ ) Real $\left(\bar{\varepsilon}_{1}\right)$ and imaginary parts $\left(\bar{\varepsilon}_{2}\right)$ of the local dielectric functions corresponding to the circles in parts $\mathrm{c}$ and $\mathrm{d}$ obtained via inversion of the finite-dipole model on a bulk medium.

to measure the amplitude $s_{\mathrm{n}}$ and phase $\phi_{\mathrm{n}}$ of the near field interferometrically (see Supporting Information for experimental details) and retrieve their spectral shapes. We reveal the characteristic spectral signature of the surface states locally by recording near-field spectra as a function of the tip position along a line across the TI surface. The topography and scattered intensity of the TI region chosen for the scan are depicted in Figure 2a. Figure $2 \mathrm{~b}$ shows a so-called hyperspectral map, where the spectral amplitude $s_{\mathrm{n}}$ is plotted as a function of frequency and position (white dashed line in Figure 2a). Here the scattered near-field amplitude is normalized to the spectral response of a silicon reference sample. The spectra measured on the $\mathrm{Si} / \mathrm{SiO}_{2}$ substrate (see vertical white line in Figure $2 \mathrm{~b}$ ) exhibit a strong phonon response at $\sim 33.8 \mathrm{THz}$, which has been reported previously. ${ }^{32,38,44}$ The spectral amplitude on the TI shows distinctly different spectral features. Two aspects are particularly noteworthy. First, there is a dominant step-like increase of the scattering response for frequencies higher than $\sim 38 \mathrm{THz}$. Second, the overall amplitude of this feature changes slightly as a function of position, in line with the variation of the scattered intensity in Figure 2a. This corroborates that local probing with near-field microscopy is crucial for revealing essential information about the local surface properties.

To investigate the depth dependence of this scattering response, we perform nanotomography. ${ }^{30,34,39-42}$ By either changing the tapping amplitude of the tip or by studying different demodulation orders, respectively, ${ }^{30,34}$ the probing volume can be altered (see Supporting Information, section 4): a large tapping amplitude probes the sample response deep into the bulk of the TI material, whereas a small tapping amplitude only interrogates the response from a shallow surface layer. ${ }^{30}$ Similarly, by investigating different demodulation orders, depth sensitivity can be achieved: higher demodulation orders $\mathrm{n}$ (for example, $\mathrm{n}=3$ ) provide shallower probing volumes as compared to smaller demodulation orders $(\mathrm{n}=2) \cdot{ }^{34}$

Representative relative amplitude and phase spectra recorded on the TI are shown in Figure 2, parts $\mathrm{c}$ and $\mathrm{d}$, respectively. Interestingly, the amplitude of the step-like feature increases for more surface-sensitive probing $(n=3$ compared to $\mathrm{n}=2$ ). Nevertheless, a direct interpretation of these spectra is quite challenging, as the scattered near fields of the coupled tip-sample system depend nontrivially on the dielectric response of the sample. In the literature, several models have been proposed to theoretically describe the nearfield response. However, these models are typically employed to calculate the scattering response starting with a complexvalued dielectric function known a priori. We have developed a numerical inversion algorithm (similar to previously demonstrated algorithms; ${ }^{34,45}$ see Supporting Information, section 3) to invert the process and directly convert the relative amplitude and phase (Figure 2c,d) of the experimentally measured scattered near-field response into the corresponding real and 

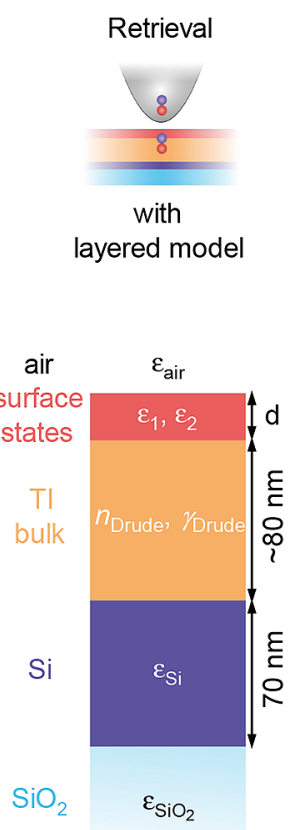

b

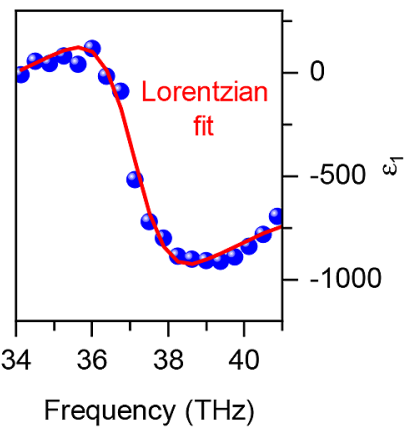

C

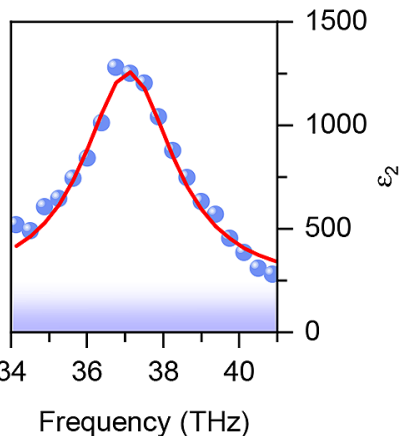

d

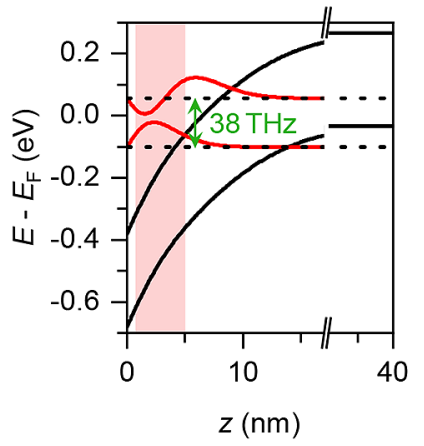

e

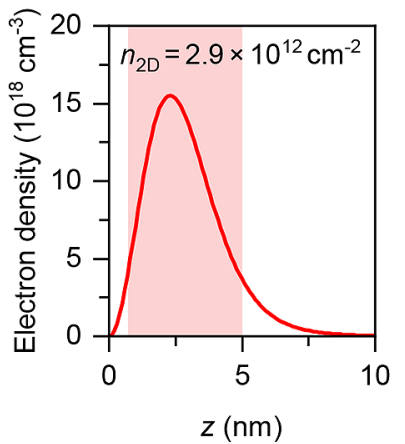

f

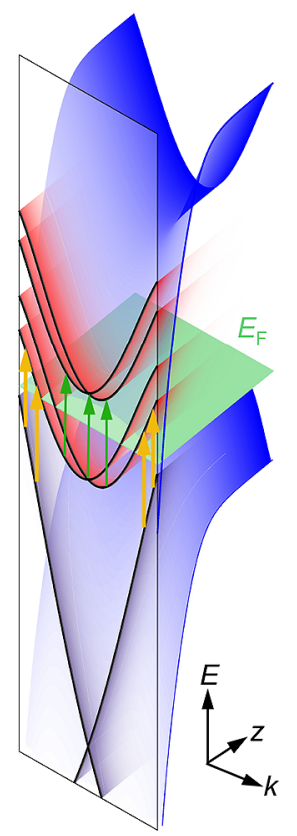

Figure 3. Retrieval of the dielectric function with a multilayer model. (a) Sketch of the layered sample structure and input parameters for the retrieval algorithm. $(\mathrm{b}, \mathrm{c})$ Real $\left(\varepsilon_{1}\right)$ and imaginary parts $\left(\varepsilon_{2}\right)$ of the local dielectric function of the surface state, obtained via numerical inversion of the finite-dipole model on a five-layer structure. The analysis was performed for $d=5 \mathrm{~nm}$. (d) Numerical simulation of the band bending of the conduction and valence band based on a self-consistent solution of the Schrödinger-Poisson equation (the simulation parameters are given in the Supporting Information). A massive, two-dimensional electron gas forms at the surface of the TI due to this quantum confinement. Green arrow: transition between the two lowest subbands, visualized via their respective wave functions (red curves). (e) Electron density close to the TI surface based on the results of the numerical band bending calculation in part $\mathrm{d}$. The shaded regions in parts $\mathrm{d}$ and e indicate the extension of the surface states. (f) Sketch of the band structure of the sample as a function of wavevector and vertical direction into the material. The TSSs (black lines) lie at the surface (vertical plane). Due to band bending of the valence and conduction bands (blue surfaces), subbands (red shaded lines) emerge at the surface. Green arrows: intersubband transitions corresponding to the measured Lorentzian-shaped dielectric function. Furthermore, transitions from the TSS to the lowest subband (orange arrows) may explain the broadband background in the dielectric function (shaded regions in Figure $2 \mathrm{f}$ and part c).

imaginary part of the local dielectric function of the material (Figure 2e,f), respectively. Our algorithm uses the finite-dipole model $^{47}$ to describe the tip-sample interaction. We verified that the qualitative features discussed in the following are also found for other models established in the literature for describing the interaction between the tip and a bulk sample (from now on referred to as "bulk models"), such as the point-dipole model ${ }^{44}$ and the lightning-rod model. ${ }^{45}$

The dielectric function of the TI sample retrieved in this way is depicted in Figure 2, parts e and f. The imaginary part of the local dielectric function (Figure $2 \mathrm{f}$ ), $\bar{\varepsilon}_{2}(\omega)$, features a sharp resonance with a Lorentzian line shape while a corresponding dispersive shape is observed in the real part, $\bar{\varepsilon}_{1}(\omega)$ (Figure 2e). Yet, the analysis with bulk models is inherently challenged since the evaluation of experimental data taken at different harmonic orders yields distinctly different dielectric functions. As seen in Figure 2, parts e and $\mathrm{f}$, the more surface-selective probing with $\mathrm{n}=3$ results in a stronger Lorentzian resonance as compared to $\mathrm{n}=2$. This inconsistency suggests that the relevant oscillator may be located at the sample surface, and a more sophisticated retrieval model inherently accounting for a depth-dependent dielectric response is necessary.

In order to disentangle the dielectric response from the bulk and the surface, we extend our description of the scattering response by combining the finite-dipole approach with a multilayer model for up to five layers, as reported by Hauer et al. ${ }^{37}$ We implement this concept in an inversion algorithm for the first time to extract the local dielectric function of the surface layer alone. Figure 3a depicts a sketch of the layer structure of our sample encompassing air, the surface states under study, the bulk TI, silicon, and silicon dioxide. The scattering response of the bulk TI is described by a Drude gas of electrons with density $n_{\text {Drude }}=6 \times 10^{18} \mathrm{~cm}^{-3}$ and damping constant $\gamma_{\text {Drude }}=25 \mathrm{THz}$ (see Supporting Information, section $4)$, taken from previous data of similar structures. ${ }^{18}$ For the silicon and silicon dioxide layers underneath, we use literature values for the dielectric functions $\varepsilon_{\mathrm{Si}}$ and $\varepsilon_{\mathrm{SiO} 2}$ (see Supporting Information). The tip radius was determined to be $36 \mathrm{~nm}$ by scanning electron microscopy and consequently set to this value for all simulations. This radius of curvature also describes the measured retraction scans with the best agreement (see Supporting Information). We make no assumptions about the dielectric properties of the surface layer under study.

In contrast to the previously employed bulk model, the multilayer approach should consistently describe the experimental scattering response for different demodulation orders (i.e., probing depths, see Supporting Information) with a single dielectric function. Yet, this ideal situation is only possible if the correct thickness $d$ of the surface states is used. In turn, the requirement to describe all experimental data with only one set 
a
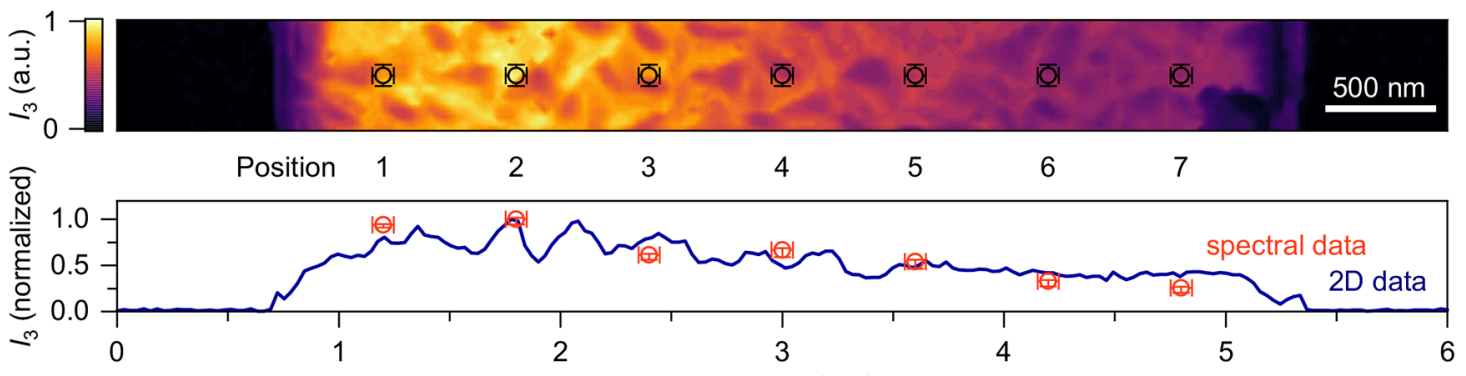

$x$ position $(\mu \mathrm{m})$

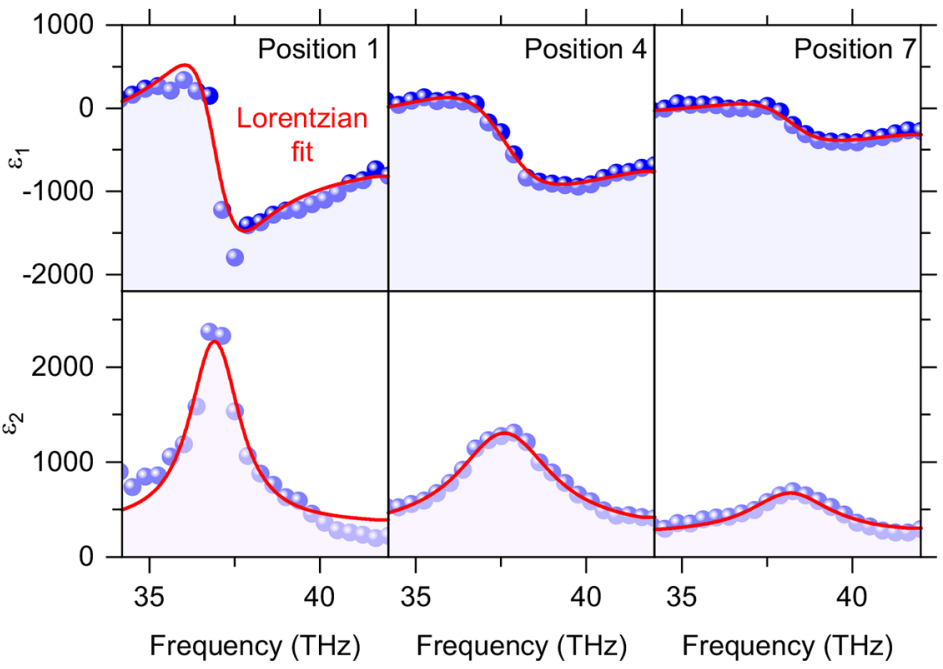

c

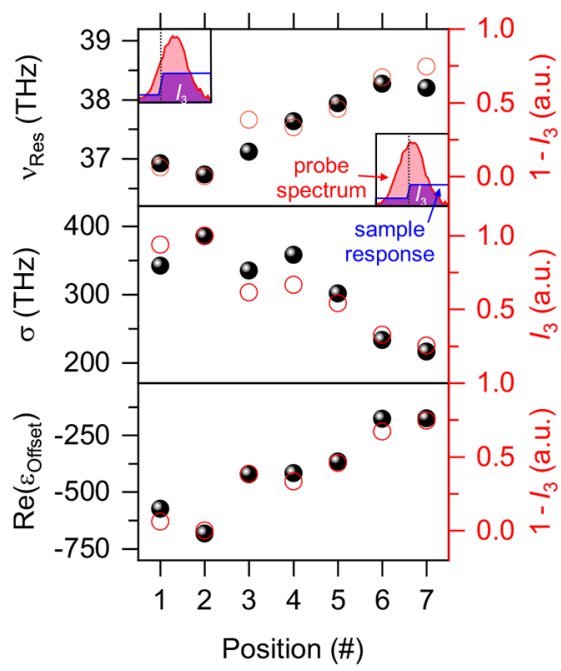

Figure 4. Tracing the nanoscale inhomogeneity of the dielectric response of the surface. (a) Top panel: Color-coded, scattered intensity $I_{3}$ of a $\left(\mathrm{Bi}_{0.5} \mathrm{Sb}_{0.5}\right)_{2} \mathrm{Te}_{3}$ structure showing nanoscale variations combined with a micron-scale gradient. Positions 1 through 7 indicate where amplitude and phase spectra are recorded. Bottom panel: Line cut of the scattered intensity $I_{3}$ taken at the vertical position in the top panel where the spectral data was acquired. For comparison, the measured amplitude spectra (at positions 1-7) can be spectrally integrated and squared. The resulting values are overlaid over the intensity curve (red circles). The error bars have been extracted from stability measurements. (b) Three representative dielectric functions of the surface states split into real $\left(\varepsilon_{1}\right.$, top panels) and imaginary parts $\left(\varepsilon_{2}\right.$, bottom panels), extracted from the spectra recorded at positions 1, 4, and 7 (blue dots). The retrieval algorithm for these measurements is equal to the one in Figure 3 . Vertically shifted Lorentzian fit curves (red curves) show excellent agreement with the data. A pronounced shift of the resonance frequency and the dielectric background is observed as a function of position. Quantitatively, the resonance frequency $\nu_{\text {Res }}$, the oscillator strength $\sigma$, and the real part of the dielectric background $\operatorname{Re}\left(\varepsilon_{\text {Offset }}\right)$ can be extracted from the fits, which are shown in part $\mathrm{c}$ as filled spheres. Interestingly, the resonance frequency and the dielectric background (from part b) depend linearly on $\left(1-I_{3}\right)$, where the scattered intensity from the bottom panel of part a was used. For the oscillator strength, we find a direct proportionality to the scattered intensity $I_{3}$. The insets illustrate the origin of this dependence of the scattered intensity $I_{3}$ (purple) being the local step-like sample response (blue) and the probe spectrum (red). In principle, this allows us to convert the full two-dimensional scan in part a, for example, into a map of local carrier density or $\mathrm{Bi}-\mathrm{Sb}$ ratio as discussed in the main text.

of parameters limits the acceptable values of $d$. In fact, we find that the new multilayer model consistently retrieves the same dielectric function for all demodulation orders and tapping amplitudes if and only if the surface layer is assumed to be only a few nanometers thick (see Supporting Information, section 4). In Figures $3 b, c$ the real and imaginary parts of the retrieved dielectric function of the surface states are shown for $d=5 \mathrm{~nm}$. In qualitative similarity to the dielectric functions obtained with the bulk models (Figure 2e,f), the exact dielectric function of the surface states is dominated by a Lorentzian resonance. Yet, the magnitudes of the real and imaginary parts of the dielectric functions $\left(\varepsilon_{1}, \varepsilon_{2}\right)$ are larger for the realistic multilayer model than for the bulk approximation, since the bulk model provides only an averaged response $\left(\bar{\varepsilon}_{1}, \bar{\varepsilon}_{2}\right)$ of the bulk and the surface.

Note that the Lorentzian resonance cannot originate from a conventional surface plasmon, since the rather low electron mobilities in bulk TIs at room temperature ${ }^{18}$ are at odds with the extremely narrow line width seen in Figure 3b,c. Instead, we will show next that the sharp absorption line as well as the transition dipole orientation (parallel to the near-field orientation and perpendicular to the surface) are characteristic of intersubband radiative transitions within a massive electron gas formed at the sample surface. Since the curvatures of subbands originating from the same conduction band are identical, optical dipole transitions between them feature a single, well-defined energy difference. Indeed, band bending, and quantum confinement of a massive 2DEG at the TI surface has already been predicted. ${ }^{21,22}$ For a quantitative picture, we calculate the effect of band bending in our particular TI alloy using a self-consistent Schrödinger-Poisson solver. ${ }^{48}$ The resulting quantum confinement of conduction band electrons perpendicular to the surface results in quantized envelope wave functions (Figure 3d) while the in-plane-momentum remains continuous, forming two-dimensional subbands. Transitions between the two subbands require the envelope wave functions of Figure $3 \mathrm{~d}$ to change, which is associated with an out-ofplane dipole moment, as observed in our experiment. These 
excitations cannot easily be observed in far-field experiments, as they lack the required out-of-plane electric field component and require evanescent near fields or grating structures to efficiently couple to the electromagnetic field.

From our numerical simulations, we find a transition energy of $157 \mathrm{meV}(\sim 38 \mathrm{THz})$ by fitting the offset between conduction band edge and Fermi energy at the surface to $0.38 \mathrm{eV}$. All remaining physical quantities are chosen according to literature values for our TI alloy (see Supporting Information). Furthermore, we compute an effective thickness for the 2DEG surface layer of $4.3 \mathrm{~nm}$ (see highlighted region in Figure $3 \mathrm{~d}, \mathrm{e})$, which is very close to the thickness $(d=5 \mathrm{~nm})$ employed in the retrieval of the dielectric function with the multilayer model in Figure 3b,c. Extensions of similar magnitude have previously been reported for massive surface states on the related material system $\mathrm{Bi}_{2} \mathrm{Se}_{3}{ }^{21}$ highlighting the potential of our technique in extracting the thickness of 2DEG or TSS layers in TI materials. The numerical calculation of the band bending also allows us to extract a maximum carrier density (Figure 3e) of $n_{3 \mathrm{D}}=1.5 \times 10^{19} \mathrm{~cm}^{-3}$ or a respective sheet density of $n_{2 \mathrm{D}}=2.9 \times 10^{12} \mathrm{~cm}^{-2}$, at the surface. This suggests that these surface states are highly conductive and contribute to the increase in scattered intensity at the surface observed experimentally.

Beside the experimentally and theoretically observed strong spectral resonance, there is a further noteworthy feature in the dielectric function. The Lorentzian line shape exhibits a nearly constant background (Figures $2 \mathrm{f}$ and $3 \mathrm{c}$, blue shaded area). The offset in the imaginary part, $\varepsilon_{2}(\omega)$, of the dielectric function is of particular interest, as it suggests an additional broadband absorption over the entire accessible spectral range. This absorption cannot stem from a direct interband transition in the TSS, as most states within the Dirac cone are occupied (Figure 3f). Instead, a possible microscopic explanation of the broadband absorption could be given by transitions between the TSS and the Rashba-split subbands ${ }^{20,21}$ (Figure 3f, orange arrows). As shown in the Supporting Information, the qualitatively different dispersion of TSS and Rashba-split subbands enables transition energies in a broad frequency range covering the probe spectrum of our experiment.

In order to harness the TSSs or 2DEG states for applications, it is imperative to investigate the effect of inhomogeneity on key parameters, such as the carrier density. With this aim, we utilize our multilayer algorithm to extract the local dielectric function at seven positions of another wedge structure on the same sample (Figure $4 \mathrm{a}$, top panel). This should allow us, in principle, to retrieve key information about carrier densities, chemical composition in alloys, and band bending on the nanoscale. The bottom panel in Figure 4a shows the line cut of the scattered intensity along these positions, as marked in the corresponding near-field image of the TI (top panel). For comparison, the obtained spectra are translated into an intensity value via spectral integration. The results are plotted alongside the line cuts in the bottom panel of Figure $4 \mathrm{a}$, where the spectral data are normalized and supplemented with error bars to account for both the lateral drift of the sample and the drift of the probe power during data acquisition.

The relative amplitude and phase spectra for each position (see Supporting Information) are then converted to their corresponding local dielectric functions, analogous to the procedure described in Figure $3 \mathrm{c}-\mathrm{f}$. Three typical dielectric functions, split into real (top panels) and imaginary parts (bottom panels), are shown in Figure 4b. Again, the sharp resonance is prominent and can be fitted with a Lorentzian line shape including a dielectric background. Strikingly, we observe a shift in resonance frequency from $\sim 36$ to $\sim 38 \mathrm{THz}$ as the tip position is varied, which is accompanied by a change in the background observed in the real part of the dielectric function. These variations can also be clearly seen in the measured spectra of the scattering response before inversion (see Supporting Information) and serve to show the degree of inhomogeneity across the sample. The extracted values of the resonance frequency, the oscillator strength, and the dielectric background are plotted in Figure $4 \mathrm{c}$ for all measured spectra as a function of the tip position and clearly highlight the variation of the dielectric function across the sample. This local variation of the intersubband resonance within the massive 2DEG at the TI surface could originate from a variety of factors. First, local fluctuations of the ratio between $\mathrm{Bi}$ and $\mathrm{Sb}$ in the ternary compound will influence the band offsets and therefore the depth of the confinement potential at the surface. Second, the dielectric constants of $\mathrm{Bi}_{2} \mathrm{Te}_{3}$ and $\mathrm{Sb}_{2} \mathrm{Te}_{3}$ differ, so the effect of band bending will vary according to alloy concentration. Furthermore, local variations of the Fermi level $^{27}$ and defects ${ }^{49}$ will also play a significant role in the local band bending and thereby the local surface carrier density.

Interestingly, both the resonance frequency and the dielectric background scale almost linearly with $\left(1-I_{3}\right)$, whereas we find a direct proportionality for the oscillator strength (see Figure 4c). The resonance frequency determines the position of the step-like feature in the scattered amplitude, whereas the dielectric background and oscillator strength govern its overall magnitude (for details see Supporting Information). Since the scattered intensity $I_{3}$ is composed of the scattering response and the probe spectrum (Figure $4 c$, insets), it shows a strong dependence on, for example, the resonance frequency. Hence, the spectrally integrated image in Figure $4 \mathrm{a}$ also reflects the spatial dependence of the local parameters of the Lorentzian-shaped resonance. Consequently, the gradient observed in the scattered intensity across the TI structure (Figures $1 \mathrm{~b}$ and $4 \mathrm{a}$ ) can be attributed to locally varying properties of the intersubband resonance (Figure 4c). This might open the way toward a conversion of the scan shown in the top panel in Figure $4 \mathrm{a}$ into a two-dimensional map of the local resonance frequency, for example, from which information about local carrier densities, $\mathrm{Bi}$ or $\mathrm{Sb}$ concentrations, Fermi level, and many more quantities could be deduced. Combining our experiments with ARPES and investigating different samples with passivated surfaces, thereby intentionally modifying the band bending, could help to unambiguously identify the TSS with SNOM.

In conclusion, we have employed near-field microscopy and tomography in the MIR to image the lateral surface inhomogeneity of selectively grown $\left(\mathrm{Bi}_{0.5} \mathrm{Sb}_{0.5}\right)_{2} \mathrm{Te}_{3}$ structures with nanoscale precision. The dominant contribution to the scattered intensity originates from a region close to the surface ( $5 \mathrm{~nm}$ thickness). By directly extracting the complex-valued dielectric function, we reveal a strong Lorentzian-shaped resonance at $\sim 38 \mathrm{THz}$ caused by an intersubband transition within the massive 2DEG, whereas an additional broadband absorption background might be an indication of the TSS. On the nanoscale, we observe local variations of the intersubband resonance, indicating lateral variations of band bending due to local fluctuations of the $\mathrm{Bi}$ or $\mathrm{Sb}$ concentration, the Fermi level 
or defects. We foresee that such a nanoscale extraction of the dielectric function using near-field microscopy will find a wide range of applications for many novel materials. In our experiments, we utilize coherent broadband spectra in the form of ultrashort pulses, ${ }^{50-52}$ setting the stage for nanoscale pump-probe experiments on topological insulators. In the future, femtosecond photoexcitation ${ }^{53-55}$ in combination with nanotomographic probing of the subsequent ultrafast dynamics should allow the interplay between various surface and bulk states to be distinguished. Complementary ultrafast nanoscale photoemission spectroscopy ${ }^{56}$ measurements could provide additional insights into the local carrier dynamics. The improved microscopic understanding will aid the development of optimized three-dimensional topological insulators with yet larger mobilities. Thus, MIR Dirac plasmons on the surfaces of TIs may ultimately be resolved and controlled in real space using ultrafast SNOM.

\section{ASSOCIATED CONTENT}

\section{S Supporting Information}

The Supporting Information is available free of charge on the ACS Publications website at DOI: 10.1021/acs.nanolett.8b03008.

Selective growth of the $\left(\mathrm{Bi}_{0.5} \mathrm{Sb}_{0.5}\right)_{2} \mathrm{Te}_{3}$ wedge structures, near-field microscopy, inversion algorithm to retrieve the nanoscale dielectric function, details of the multilayer scattering model, retrieved surface dielectric functions across the wedge, numerical simulations of the band bending at the TI surface, and origin of the broadband dielectric background (PDF)

\section{AUTHOR INFORMATION}

\section{Corresponding Authors}

*(M.A.H.) E-mail: markus.huber@ur.de. Fax: +49 941943 4223.

*(R.H.) E-mail: rupert.huber@ur.de. Fax: +49 9419434223.

\section{ORCID $\odot$}

Fabian Mooshammer: 0000-0002-9744-2694

Jessica L. Boland: 0000-0002-6351-5699

\section{Author Contributions}

F.M., F.S., M.A.H., M.Z., H.W., M.P., J.L.B, T.L.C., and R.H. performed the experiments. C.W., M.L., J.K., G.M., and D.G. designed, grew and characterized the topological insulator structures. The numerical simulations of the band bending were done by F.M., F.S., M.A.H., and J.L.B.. F.M. and M.A.H. implemented the algorithm for the retrieval of the dielectric function. All authors contributed to the discussions of the results. The manuscript was written by F.M., F.S., M.A.H, J.L.B., T.L.C., and R.H. with input from all authors.

Notes

The authors declare no competing financial interest.

\section{ACKNOWLEDGMENTS}

The authors thank Simon Maier for fruitful discussions. The work in Regensburg was supported by the European Research Council through Grant Number 305003 (QUANTUMsubCYCLE) as well as by the Deutsche Forschungsgemeinschaft (through grant numbers HU1598/3 \& CO1492, SFB 1277 Project A05, and GRK 1570). The work in Jülich was supported by the Helmholtz Virtual Institute for Topological Insulators (Jülich-Aachen-Würzburg-Shanghai) and the
DFG-funded Priority Program SPP1666. J.L.B. acknowledges the support of the A. v. Humboldt Foundation.

\section{REFERENCES}

(1) Moore, J. E. The birth of topological insulators. Nature 2010, 464, 194-198.

(2) Hasan, M. Z.; Kane, C. L. Colloquium: Topological insulators. Rev. Mod. Phys. 2010, 82, 3045-3067.

(3) Zhang, H.; Liu, C.-X.; Qi, X.-L.; Dai, X.; Fang, Z.; Zhang, S.-C. Topological insulators in $\mathrm{Bi}_{2} \mathrm{Se}_{3}, \mathrm{Bi}_{2} \mathrm{Te}_{3}$ and $\mathrm{Sb}_{2} \mathrm{Te}_{3}$ with a single Dirac cone on the surface. Nat. Phys. 2009, 5, 438-442.

(4) Chen, Y. L.; Analytis, J. G.; Chu, J.-H.; Liu, Z. K.; Mo, S.-K.; Qi, X. L.; Zhang, H. J.; Lu, D. H.; Dai, X.; Fang, Z.; Zhang, S. C.; Fisher, I. R.; Hussain, Z.; Shen, Z.-X. Experimental Realization of a ThreeDimensional Topological Insulator, $\mathrm{Bi}_{2} \mathrm{Te}_{3}$. Science 2009, 325, 178181.

(5) Ando, Y. Topological Insulator Materials. J. Phys. Soc. Jpn. 2013, 82, 102001.

(6) Politano, A.; Viti, L.; Vitiello, M. S. Optoelectronic devices, plasmonics, and photonics with topological insulators. APL Mater. 2017, 5, 035504.

(7) McIver, J. W.; Hsieh, D.; Steinberg, H.; Jarillo-Herrero, P.; Gedik, N. Control over topological insulator photocurrents with light polarization. Nat. Nanotechnol. 2012, 7, 96-100.

(8) Braun, L.; Mussler, G.; Hruban, A.; Konczykowski, M.; Schumann, T.; Wolf, M.; Münzenberg, M.; Perfetti, L.; Kampfrath, $\mathrm{T}$. Ultrafast photocurrents at the surface of the three-dimensional topological insulator $\mathrm{Bi}_{2} \mathrm{Se}_{3}$. Nat. Commun. 2016, 7, 13259.

(9) Kuroda, K.; Reimann, J.; Güdde, J.; Höfer, U. Generation of Transient Photocurrents in the Topological Surface State of $\mathrm{Sb}_{2} \mathrm{Te}_{3}$ by Direct Optical Excitation with Midinfrared Pulses. Phys. Rev. Lett. 2016, 116, 076801.

(10) Kastl, C.; Karnetzky, C.; Karl, H.; Holleitner, A. W. Ultrafast helicity control of surface currents in topological insulators with nearunity fidelity. Nat. Commun. 2015, 6, 6617.

(11) Reimann, J.; Schlauderer, S.; Schmid, C. P.; Langer, F.; Baierl, S.; Kokh, K. A.; Tereshchenko, O. E.; Kimura, A.; Lange, C.; Güdde, J.; Höfer, U.; Huber, R. Subcycle observation of lightwave-driven Dirac currents in a topological surface band. Nature 2018, 562, 396400.

(12) Wang, Y. H.; Steinberg, H.; Jarillo-Herrero, P.; Gedik, N. Observation of Floquet-Bloch States on the Surface of a Topological Insulator. Science 2013, 342, 453-457.

(13) Mahmood, F.; Chan, C.-K.; Alpichshev, Z.; Gardner, D.; Lee, Y.; Lee, P. A.; Gedik, N. Selective scattering between Floquet-Bloch and Volkov states in a topological insulator. Nat. Phys. 2016, 12, 306310 .

(14) Di Pietro, P.; Ortolani, M.; Limaj, O.; Di Gaspare, A.; Giliberti, V.; Giorgianni, F.; Brahlek, M.; Bansal, N.; Koirala, N.; Oh, S.; Calvani, P.; Lupi, S. Observation of Dirac plasmons in a topological insulator. Nat. Nanotechnol. 2013, 8, 556-560.

(15) Giorgianni, F.; Chiadroni, E.; Rovere, A.; Cestelli-Guidi, M.; Perucchi, A.; Bellaveglia, M.; Castellano, M.; Di Giovenale, D.; Di Pirro, G.; Ferrario, M.; Pompili, R.; Vaccarezza, C.; Villa, F.; Cianchi, A.; Mostacci, A.; Petrarca, M.; Brahlek, M.; Koirala, N.; Oh, S.; Lupi, S. Strong nonlinear terahertz response induced by Dirac surface states in $\mathrm{Bi}_{2} \mathrm{Se}_{3}$ topological insulator. Nat. Commun. 2016, 7, 11421.

(16) Zhang, J.; Chang, C.-Z.; Zhang, Z.; Wen, J.; Feng, X.; Li, K.; Liu, M.; He, K.; Wang, L.; Chen, X.; Xue, Q.-K.; Ma, X.; Wang, Y. Band structure engineering in $\left(\mathrm{Bi}_{1-\mathrm{x}} \mathrm{Sb}_{\mathrm{x}}\right)_{2} \mathrm{Te}_{3}$ ternary topological insulators. Nat. Commun. 2011, 2, 574.

(17) Kellner, J.; Eschbach, M.; Kampmeier, J.; Lanius, M.; Mlynczak, E.; Mussler, G.; Holländer, B.; Plucinski, L.; Liebmann, M.; Grützmacher, D.; Schneider, C. M.; Morgenstern, M. Tuning the Dirac point to the Fermi level in the ternary topological insulator $\left(\mathrm{Bi}_{1-\mathrm{x}} \mathrm{Sb}_{\mathrm{x}}\right)_{2} \mathrm{Te}_{3}$. Appl. Phys. Lett. 2015, 107, 251603.

(18) Weyrich, C.; Drögeler, M.; Kampmeier, J.; Eschbach, M.; Mussler, G.; Merzenich, T.; Stoica, T.; Batov, I. E.; Schubert, J.; Plucinski, L.; Beschoten, B.; Schneider, C. M.; Stampfer, C.; 
Grützmacher, D.; Schäpers, Th. Growth, characterization, and transport properties of ternary $\left(\mathrm{Bi}_{1-\mathrm{x}} \mathrm{Sb}_{\mathrm{x}}\right)_{2} \mathrm{Te}_{3}$ topological insulator layers. J. Phys.: Condens. Matter 2016, 28, 495501.

(19) Green, A. J.; Dey, S.; An, Y. Q.; O'Brien, B.; O'Mullane, S.; Thiel, B.; Diebold, A. C. Surface oxidation of the topological insulator $\mathrm{Bi}_{2} \mathrm{Se}_{3}$. J. Vac. Sci. Technol., A 2016, 34, 061403.

(20) Bianchi, M.; Guan, D.; Bao, S.; Mi, J.; Iversen, B. B.; King, P. D. C.; Hofmann, P. Coexistence of the topological state and a twodimensional electron gas on the surface of $\mathrm{Bi}_{2} \mathrm{Se}_{3}$. Nat. Commun. 2010, 1, 128.

(21) Bahramy, M. S.; King, P. D. C.; de la Torre, A.; Chang, J.; Shi, M.; Patthey, L.; Balakrishnan, G.; Hofmann, Ph.; Arita, R.; Nagaosa, N.; Baumberger, F. Emergent quantum confinement at topological insulator surfaces. Nat. Commun. 2012, 3, 1159.

(22) Eschbach, M.; Młyńczak, E.; Kellner, J.; Kampmeier, J.; Lanius, M.; Neumann, E.; Weyrich, C.; Gehlmann, M.; Gospodarič, P.; Döring, S.; Mussler, G.; Demarina, N.; Luysberg, M.; Bihlmayer, G.; Schäpers, T.; Plucinski, L.; Blügel, S.; Morgenstern, M.; Schneider, C. M.; Grützmacher, D. Realization of a vertical topological $\mathrm{p}-\mathrm{n}$ junction in epitaxial $\mathrm{Sb}_{2} \mathrm{Te}_{3} / \mathrm{Bi}_{2} \mathrm{Te}_{3}$ heterostructures. Nat. Commun. 2015, 6, 8816.

(23) Zhang, Y.; He, K.; Chang, C.-Z.; Song, C.-L.; Wang, L.; Chen, X.; Jia, J.; Fang, Z.; Dai, X.; Shan, W.-Y.; Shen, S.-Q.; Niu, Q.; Qi, X.; Zhang, S.-C.; Ma, X.; Xue, Q.-K. Crossover of the three-dimensional topological insulator $\mathrm{Bi}_{2} \mathrm{Se}_{3}$ to the two-dimensional limit. Nat. Phys. 2010, 6, 584-588.

(24) Sobota, J. A.; Yang, S.; Analytis, J. G.; Chen, Y. L.; Fisher, I. R.; Kirchmann, P. S.; Shen, Z.-X. Ultrafast Optical Excitation of a Persistent Surface-State Population in the Topological Insulator $\mathrm{Bi}_{2} \mathrm{Se}_{3}$. Phys. Rev. Lett. 2012, 108, 117403.

(25) Borisova, S.; Krumrain, J.; Luysberg, M.; Mussler, G.; Grützmacher, D. Mode of Growth of Ultrathin Topological Insulator $\mathrm{Bi}_{2} \mathrm{Te}_{3}$ Films on Si (111) Substrates. Cryst. Growth Des. 2012, 12, 6098-6103.

(26) Macedo, R. J.; Harrison, S. E.; Dorofeeva, T. S.; Harris, J. S.; Kiehl, R. A. Nanoscale Probing of Local Electrical Characteristics on MBE-Grown $\mathrm{Bi}_{2} \mathrm{Te}_{3}$ Surfaces under Ambient Conditions. Nano Lett. 2015, 15, 4241-4247.

(27) Knispel, T.; Jolie, W.; Borgwardt, N.; Lux, J.; Wang, Z.; Ando, Y.; Rosch, A.; Michely, T.; Grüninger, M. Charge puddles in the bulk and on the surface of the topological insulator $\mathrm{BiSbTeSe}_{2}$ studied by scanning tunneling microscopy and optical spectroscopy. Phys. Rev. B: Condens. Matter Mater. Phys. 2017, 96, 195135.

(28) Hauer, B.; Saltzmann, T.; Simon, U.; Taubner, T. Solvothermally Synthesized $\mathrm{Sb}_{2} \mathrm{Te}_{3}$ Platelets Show Unexpected Optical Contrasts in Mid-Infrared Near-Field Scanning Microscopy. Nano Lett. 2015, 15, 2787-2793.

(29) Hillenbrand, R.; Taubner, T.; Keilmann, F. Phonon-enhanced light-matter interaction at the nanometre scale. Nature 2002, 418, $159-162$.

(30) Eisele, M.; Cocker, T. L.; Huber, M. A.; Plankl, M.; Viti, L.; Ercolani, D.; Sorba, L.; Vitiello, M. S.; Huber, R. Ultrafast multiterahertz nano-spectroscopy with sub-cycle temporal resolution. Nat. Photonics 2014, 8, 841-845.

(31) Huber, M. A.; Plankl, M.; Eisele, M.; Marvel, R. E.; Sandner, F.; Korn, T.; Schüller, C.; Haglund, R. F., Jr.; Huber, R.; Cocker, T. L. Ultrafast Mid-Infrared Nanoscopy of Strained Vanadium Dioxide Nanobeams. Nano Lett. 2016, 16, 1421-1427.

(32) Huber, M. A.; Mooshammer, F.; Plankl, M.; Viti, L.; Sandner, F.; Kastner, L. Z.; Frank, T.; Fabian, J.; Vitiello, M. S.; Cocker, T. L.; Huber, R. Femtosecond photo-switching of interface polaritons in black phosphorus heterostructures. Nat. Nanotechnol. 2017, 12, 207211.

(33) McLeod, A. S.; van Heumen, E.; Ramirez, J. G.; Wang, S.; Saerbeck, T.; Guenon, S.; Goldflam, M.; Anderegg, L.; Kelly, P.; Mueller, A.; Liu, M. K.; Schuller, I. K.; Basov, D. N. Nanotextured phase coexistence in the correlated insulator $\mathrm{V}_{2} \mathrm{O}_{3}$. Nat. Phys. 2016, 13, 80-86.
(34) Govyadinov, A. A.; Mastel, S.; Golmar, F.; Chuvilin, A.; Carney, P. S.; Hillenbrand, R. Recovery of Permittivity and Depth from NearField Data as a Step toward Infrared Nanotomography. ACS Nano 2014, 8, 6911-6921.

(35) Kampmeier, J.; Weyrich, C.; Lanius, M.; Schall, M.; Neumann, E.; Mussler, G.; Schäpers, T.; Grützmacher, D. Selective area growth of $\mathrm{Bi}_{2} \mathrm{Te}_{3}$ and $\mathrm{Sb}_{2} \mathrm{Te}_{3}$ topological insulator thin films. J. Cryst. Growth 2016, 443, 38-42.

(36) Klarskov, P.; Kim, H.; Colvin, V. L.; Mittleman, D. M. Nanoscale Laser Terahertz Emission Microscopy. ACS Photonics 2017, 4, 2676-2680.

(37) Hauer, B.; Engelhardt, A. P.; Taubner, T. Quasi-analytical model for scattering infrared near-field microscopy on layered systems. Opt. Express 2012, 20, 13173.

(38) Krutokhvostov, R.; Govyadinov, A. A.; Stiegler, J. M.; Huth, F.; Chuvilin, A.; Carney, P. S.; Hillenbrand, R. Enhanced resolution in subsurface near-field optical microscopy. Opt. Express 2012, 20, 593.

(39) Moon, K.; Park, H.; Kim, J.; Do, Y.; Lee, S.; Lee, G.; Kang, H.; Han, H. Subsurface Nanoimaging by Broadband Terahertz Pulse Near-Field Microscopy. Nano Lett. 2015, 15, 549-552.

(40) Taubner, T.; Keilmann, F.; Hillenbrand, R. Nanoscale-resolved subsurface imaging by scattering-type near-field optical microscopy. Opt. Express 2005, 13, 8893.

(41) Raschke, M. B.; Lienau, C. Apertureless near-field optical microscopy: Tip-sample coupling in elastic light scattering. Appl. Phys. Lett. 2003, 83, 5089-5091.

(42) Zhang, L. M.; Andreev, G. O.; Fei, Z.; McLeod, A. S.; Dominguez, G.; Thiemens, M.; Castro-Neto, A. H.; Basov, D. N.; Fogler, M. M. Near-field spectroscopy of silicon dioxide thin films. Phys. Rev. B: Condens. Matter Mater. Phys. 2012, 85, 075419.

(43) Taubner, T.; Keilmann, F.; Hillenbrand, R. Effect of Tip Modulation on Image Contrast in Scattering-Type Near-Field Optical Microscopy. J. Kor. Phys. Soc. 2005, 47, 213-216.

(44) Fei, Z.; Andreev, G. O.; Bao, W.; Zhang, L. M.; McLeod, A. S.; Wang, C.; Stewart, M. K.; Zhao, Z.; Dominguez, G.; Thiemens, M.; Fogler, M. M.; Tauber, M. J.; Castro-Neto, A. J.; Lau, C. N.; Keilmann, F.; Basov, D. N. Infrared Nanoscopy of Dirac Plasmons at the Graphene- $\mathrm{SiO}_{2}$ Interface. Nano Lett. 2011, 11, 4701-4705.

(45) McLeod, A. S.; Kelly, P.; Goldflam, M. D.; Gainsforth, Z.; Westphal, A. J.; Dominguez, G.; Thiemens, M. H.; Fogler, M. M.; Basov, D. N. Model for quantitative tip-enhanced spectroscopy and the extraction of nanoscale-resolved optical constants. Phys. Rev. B: Condens. Matter Mater. Phys. 2014, 90, 85136.

(46) Knoll, B.; Keilmann, F. Near-field probing of vibrational absorption for chemical microscopy. Nature 1999, 399, 134-137.

(47) Cvitkovic, A.; Ocelic, N.; Hillenbrand, R. Analytical model for quantitative prediction of material contrasts in scattering-type nearfield optical microscopy. Opt. Express 2007, 15, 8550.

(48) Birner, S.; Zibold, T.; Andlauer, T.; Kubis, T.; Sabathil, M.; Trellakis, A.; Vogl, P. nextnano: General Purpose 3-D Simulations. IEEE Trans. Electron Devices 2007, 54, 2137-2142.

(49) Urazhdin, S.; Bilc, D.; Tessmer, S. H.; Mahanti, S. D.; Kyratsi, T.; Kanatzidis, M. G. Scanning tunneling microscopy of defect states in the semiconductor $\mathrm{Bi}_{2} \mathrm{Se}_{3}$. Phys. Rev. B: Condens. Matter Mater. Phys. 2002, 66, 161306.

(50) Ulbricht, R.; Hendry, E.; Shan, J.; Heinz, T. F.; Bonn, M. Carrier dynamics in semiconductors studied with time-resolved terahertz spectroscopy. Rev. Mod. Phys. 2011, 83, 543-586.

(51) Jepsen, P. U.; Cooke, D. G.; Koch, M. Terahertz spectroscopy and imaging - Modern techniques and applications. Laser Photon. Rev. 2011, 5, 124-166.

(52) Dhillon, S. S.; Vitiello, M. S.; Linfield, E. H.; Davies, A. G.; Hoffmann, M. C.; Booske, J.; Paoloni, C.; Gensch, M.; Weightman, P.; Williams, G. P.; Castro-Camus, E.; Cumming, D. R. S.; Simoens, F.; Escorcia-Carranza, I.; Grant, J.; Lucyszyn, S.; Kuwata-Gonokami, M.; Konishi, K.; Koch, M.; Schmuttenmaer, C. A.; Cocker, T. L.; Huber, R.; Markelz, A. G.; Taylor, Z. D.; Wallace, V. P.; Zeitler, J. A.; Sibik, J.; Korter, T. M.; Ellison, B.; Rea, S.; Goldsmith, P.; Cooper, K. B.; Appleby, R.; Pardo, D.; Huggard, P. G.; Krozer, V.; Shams, H.; 
Fice, M.; Renaud, C.; Seeds, A.; Stöhr, A.; Naftaly, M.; Ridler, N.; Clarke, R.; Cunningham, J. E.; Johnston, M. B. The 2017 terahertz science and technology roadmap. J. Phys. D: Appl. Phys. 2017, 50, No. 043001.

(53) Luo, C. W.; Wang, H. J.; Ku, S. A.; Chen, H.-J.; Yeh, T. T.; Lin, J.-Y.; Wu, K. H.; Juang, J. Y.; Young, B. L.; Kobayashi, T.; Cheng, C.M.; Chen, C.-H.; Tsuei, K.-D.; Sankar, R.; Chou, F. C.; Kokh, K. A.; Tereshchenko, O. E.; Chulkov, E. V.; Andreev, Yu. M.; Gu, G. D. Snapshots of Dirac Fermions near the Dirac Point in Topological Insulators. Nano Lett. 2013, 13, 5797-5802.

(54) Reimann, J.; Güdde, J.; Kuroda, K.; Chulkov, E. V.; Höfer, U. Spectroscopy and dynamics of unoccupied electronic states of the topological insulators $\mathrm{Sb}_{2} \mathrm{Te}_{3}$ and $\mathrm{Sb}_{2} \mathrm{Te}_{2} \mathrm{~S}$. Phys. Rev. B: Condens. Matter Mater. Phys. 2014, 90, 081106.

(55) Sobota, J. A.; Yang, S. L.; Kemper, A. F.; Lee, J. J.; Schmitt, F. T.; Li, W.; Moore, R. G.; Analytis, J. G.; Fisher, I. R.; Kirchmann, P. S.; Devereaux, T. P.; Shen, Z. X. Direct Optical Coupling to an Unoccupied Dirac Surface State in the Topological Insulator $\mathrm{Bi}_{2} \mathrm{Se}_{3}$. Phys. Rev. Lett. 2013, 111, 136802.

(56) Man, M. K. L.; Margiolakis, A.; Deckoff-Jones, S.; Harada, T.; Wong, E. L.; Krishna, M. B.; Madéo, J.; Winchester, A.; Lei, S.; Vajtai, R.; Ajayan, P. M.; Dani, K. M. Imaging the motion of electrons across semiconductor heterojunctions. Nat. Nanotechnol. 2017, 12, 36-40. 